\title{
Review of Control Mechanism of Multi-fingered Robotic Arm and Proposal of New Design
}

\author{
Anand Kumar Kirori* Prof. Rajeshwar Lal Dua \\ M.Tech Scholar Head of DepartmentDepartment of Electronic \& Communication engineering JNU Jaipur
}

\begin{abstract}
From the last two decades a large amount of efforts have been made to develop and control anthropomorphic dexterous multi-fingered robotic hand which can replicate the behaviour of a real human hand using multiple joints as DOF and controlling mechanism like Master-Slave. In this paper, after an initial description of the control mechanism and complexity of the design of a robot hand, we analyze the different approaches and structure of some important works carried out by the researchers all around the world. To control a robotic hand, either the master-slave technique is used or vision based object manipulation is used to grab the object. Here, we will see the different parameters like performance, precision, distance of operation, complexity, etc in the previous work done by the researchers. We then continue by presenting a new design to control the robot hand and to increase the ability to operate the robot hand in real-time changing environment.
\end{abstract}

Keywords: Multi-fingered, Robotic arm, Robot hand, Dexterous manipulation

\section{INTRODUCTION}

The human hand is one of the most versatile and complex organ of the human body capable of performing wide range of tasks and comprises of different configurations; thus, the subject has become more interesting to construct similar performing anthropomorphic robotic designs. It can not only be implemented in medical science, but also can be very useful in engineering field. Robotic hand which has been proposed in the [1] and [2] resembles with the human like structure and the capability to manipulate and grasp objects. However, the versatility and the level of complexity of robotic hand are directly proportional to each other; that is, with the increase in versatility, the complexity of the design increases at the similar level. On the other hand, when it comes to increase the degree of freedom (DOFs) of the robotic hand, the more complex algorithm is required to develop in order to control the functioning of robot and to achieve the high precision level.

The inspiration for the grasping and manipulating objects can not only be drawn from the hardware of the natural systems like human and animals, but also require study of processing of signals and transmission of signals from brain to hand in order to understand how the arm is controlled actually by cerebral system.

In the following sections we will study and analyze the realtime controlling of different hand models developed in the engineering field attempting to replicate the behavior of human hand.

\section{REVIEW OF ROBOTIC HANDS}

\section{A. Handroid}

In order to replicate the human hand behaviour and functionality, the ITK developed a five-fingered robotic hand and named it "Handroid". The "Handroid" is a remotely-operable five-finger movable robot hand which can Be used in engineering environments which are inaccessible or dangerous to human hands [1]. This design consists of four fingers and a thumb which mimics the human hand design and consists of 15 Degree Of Freedom (DOF) which is equal to the number of a real human hand. The motion of this robotic hand is not restricted and can easily perform the movements which a real human hand can do.

This robot hand is designed and control in such a way that it replicates the operation performed by the master. ITK has developed a glove-type controller that remotely controls the Handroid by mimicking the operators hand movements [1]. In this design all the five fingers are made up of light weight aircraft parts and include the advance metalworking techniques. The goal of this anthropomorphic robotic hand is to perform the grasping tasks and to work in the dynamically changing environment which can be dangerous for humans. This design includes slave as robot hand and master as operator, wearing the glove. When user performs any movements, the sensors built in the glove senses the motion performed and sends the calculated signal to the controller connected to the slave. This mechanism provides the realtime controlling and movement of the robot hand with respective to the operator's hand. 6 motors are connected to the wrist of the robot hand in which 4 are used to control the 4 fingers and 2 motors are used to control the thumb in vertical and horizontal direction to provide the $3 \mathrm{D}$ motion. It also includes the fluid motion to provide the better control. Each phalanx cannot be controlled individually because there are linkages and are connected in such a way to resembles to the real human hand and can provide the similar motions of human hand while grasping any object. 


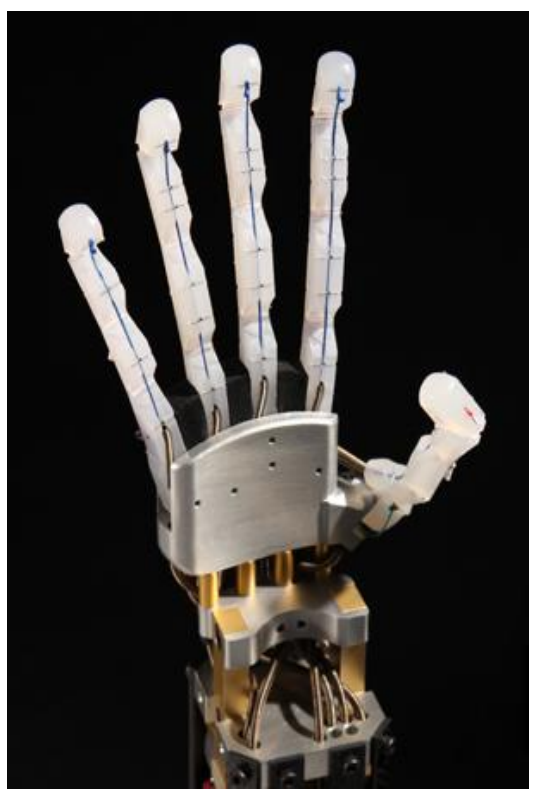

Fig 1 "Handroid"

\section{B. The Shadow EDC Hand}

It is developed by the Shadow Robot Company, in Fig 2, is considered to be the most advanced Dextrous Hands developed in the world. The new Shadow EtherCAT Dual CAN (EDC) hand sees a sizeable increase in performance for the world leading Shadow Dexterous Hand [3].

This design provides 24 degrees of freedom which can be further divided as 4 DOF each for index, middle and ring finger, $5 \mathrm{DOF}$ each for little finger and thumb including 2 DOF for wrist. It has integrated sensing and positioning control mechanism which later results into precise control of robotic hand by off-board controller or an integrated platform.

Basically, two types of Shadow EDC hand can be found as Pneumatic and Motorised that is, based on the control mechanism. In Pneumatic Shadow hand, the movement of the fingers are controller by air muscles [4]. This type of hand includes an integrated bank of 40 air muscles which is being used to provide a complete motion to the hand. These air muscles can be used to grasp soft and fragile objects. Motorised Shadow hand consists of smart motors which are being used to hold an object.

This hand can be fitted with additional sensors like touch sensors, pressure sensors on the fingertips to offer high precision feedback and better sensitivity to detect the small objects.

It includes number of collection of modules for providing analogue sensing, controlling the solenoid valves, controlling the motor and encoders setup on shaft and also for other purposes. It is controlled by the software developed in $\mathrm{C}$ language, where algorithms are implemented in 8-bit microcontroller which is then migrated to Embedded Controller implemented on-board.

A 2-joint wrist is also implemented for providing the complete motion of robot hand similar to that of human wrist and fingers.

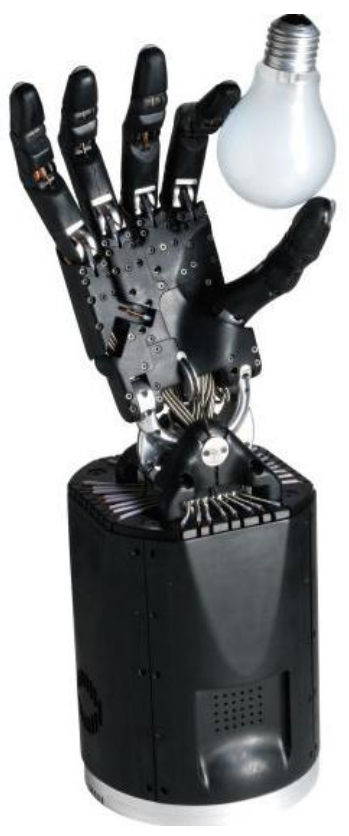

Fig 2 Shadow C6M Smart Motor Hand

\section{SBC Hand}

Kyu-Jin Cho and Harry Asada from MIT developed a SBC Hand in Fig 3, to demonstrate a lightweight robotic hand which uses an SMA actuator array [5]. This hand uses the artificial muscle actuators since they are light in weight and compact in size and delivers large power to weight ratio. Controlling mechanism of actuators in this design is divided into co-activated group of segments which is then controlled using simple ON-OFF for each segment.

The concept of segments is applied to reduce the complexity of control [5]. This hand is consists of 16 actuators which is controlled by $8 \mathrm{C}$-segments to provide the 256 distinct postures.

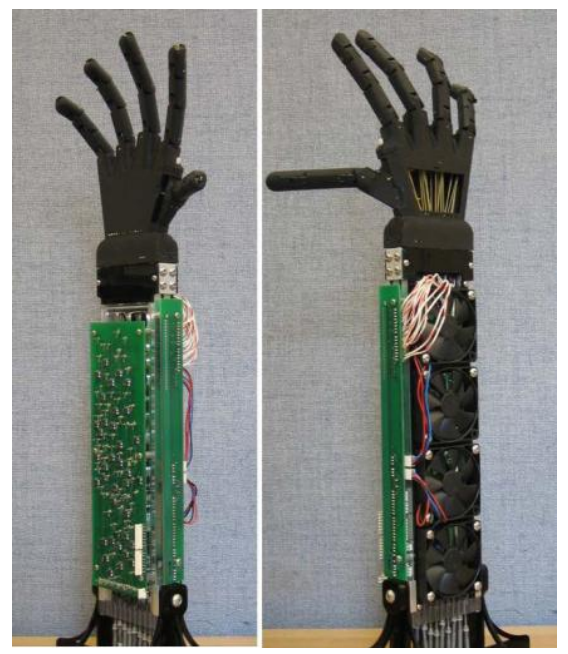

Fig 3 SBC Hand

Different hand postures were gathered on the basis of human hand movement in everyday life. These postures were 16 in number and used to generate the segments which are controller with the help of cyberglove which is used by the 
operator to control the slave (robot hand). Each finger in this design mimics the movement of human hand with the help of segments and cyberglove.

\section{D. iLimb}

ILimb (Fig 4) is a prosthetic hand which looks and provides movements like a real human hand. In this design, each finger moves independently and bends at the natural joints so that it can accurately adapt to fit around the shape of the object you want to grasp [6]. It is controlled by software called biosim used to select from the wide range of automated grips and gesture for gripping the objects with high precision. The hand automatically moves to the natural position after certain period of inactivity [7].

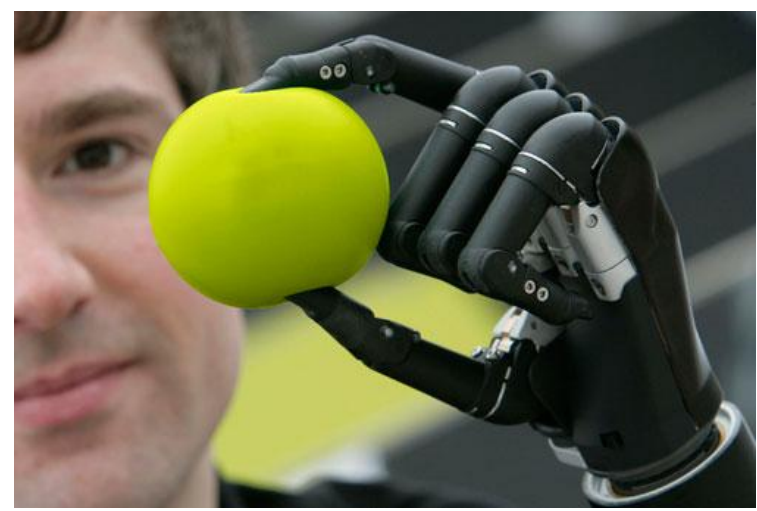

Fig 4 iLimb

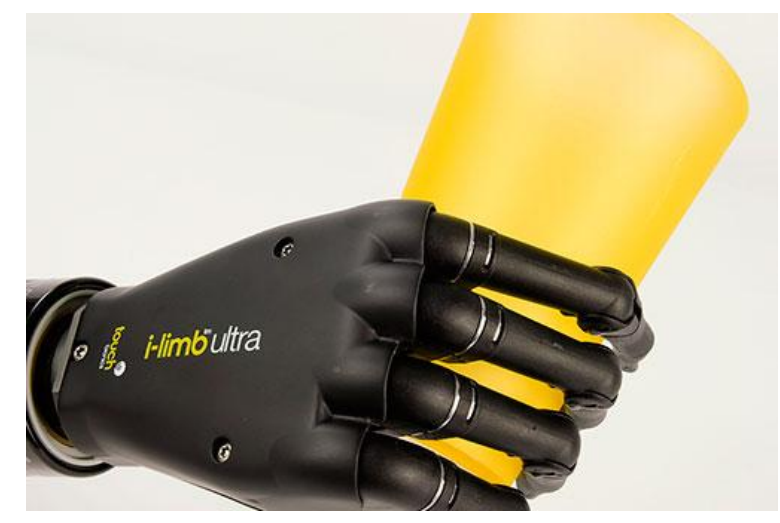

Fig 5 iLimb holding a glass

This robot hand consists of 5 individually powered fingers where the thumb can be rotatable manually to adjust the grasping position. It also include manually rotatable wrist which can be integrated with an electronic wrist rotator. By default, the software includes 13 different hand gestures which are commonly used by human hand in daily use like holding a ball using finger and thumb, holding a glass using all five fingers.

The control mechanism of this hand is based on the varying input signals by the software based on the user settings, more strong the input signal, faster the movement of fingers. It is light in weight and possesses strong durability because of the aluminium chassis used for hardware design. The software used provides the flexibility to user to create custom gestures according to their needs. It is available in two colours and medium and small size. One of its features includes the auto grasp which prevents the slipping of the object.

\section{E. Kinetic Humanoid}

KH (Kinetic Humanoid) is presented by Tetsuya Mouri and Haruhisa Kawasaki as an Anthropomorphic Robot Hand which includes Master-Slave system for tele-operation. Fig. 6 represents the robot hand where the shape and movements are replication of that to real human hand. This hand can not only be utilized in holding and dexterous manipulation of objects but can also be used as communication device using sign language. The robot hand grasps and manipulates the objects dexterously with the help of tactile sensors and force sensors in the fingers [8].

The size of this hand is larger than that of human because of high output motors which is being used. To hold the objects a PC-based master slave system was constructed where the operator is the Master and the robot is the slave. The master uses a force feedback glove [9] to generate the force while grasping the objects which can then be transferred to the human hand by the torque produced by servomotor at the finger tips of human hand connected through a wire rope.

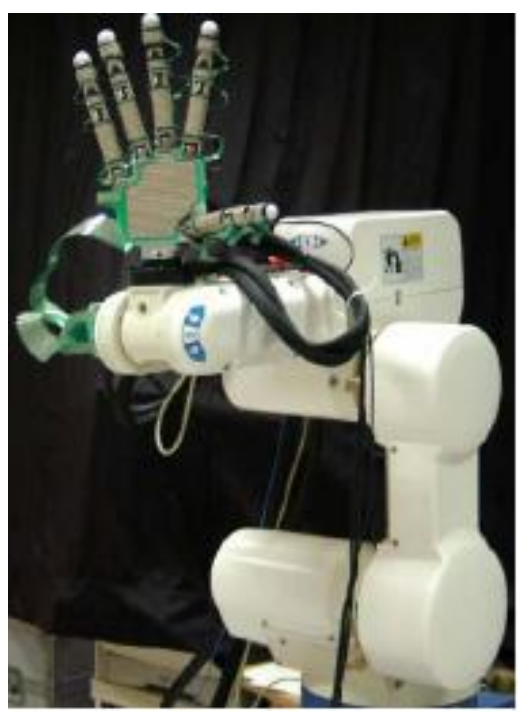

Fig 6 Kinetic Humanoid

This design consists of 20 joints and 15 degrees of freedom motioned by servomotors and of weight $0.656 \mathrm{~kg}$ with high velocity control which provides faster movements of fingers than human fingers.

\section{CONCLUSION}

From the study of the above robot hands developed by many researchers, we can say that technology has been growing at a faster pace to mimic the human hand in order to perform various tasks with high precision. We observed that most of the robot hands are either controlled by the gloves used by operator like cyberglove, force-feedback glove, etc or by the software installed on PC with some default set of positions/gestures to grasp or manipulate the objects. 
After the analysis of the above various robot hands, we found that they have limited distance of operation for controlling the slave and contains much complexity in the design of control mechanism. Here, authors would like to propose a new design using the controlling of multi-fingered robot hand using the human gestures/movement using Image Processing and transmission of generated signals using the Internet Protocols where there will be no limitation on distance of operation.

In the proposed design of robotic hand with five fingers, each finger will have 3 degrees of freedoms to be controlled by micro-servos. These micro-servos will be operated by onboard controller which will generate pulses based on the real-time movement of actual human hand, captured by 2dimensional image capturing devices and the transmission of these signals from client to server will be on IPv6 layer with 20 frames per second approx. High precision and real-time processing algorithm will be designed by authors to provide real-time operation of Robotic Hand in dynamically changing environment.

\section{REFERENCES}

[1] ITK, Multi-fingered Robot Hands Handroid from: http://www.itk-pro.com/en/pro/kindengisyu.htm

[2] Touch Bionics, i-limb-ultra from http://www.touchbionics.com/products/activeprostheses/i-limb-ultra/

[3] Shadow Robot Company: http://www.shadowrobot.com/hand/overview.shtml

[4] Y. Sakaguchi, T.Takagi, Y. Imamura, Bridgestone Corporation, Actuator, European Patent Office patent EP 0163370 A1, 1985

[5] Kyu-Jin Cho "SBC Hand: A lightweight Robotic Hand with an SMA Actuator Array Implementing $C$ segmentation" IEEE International Conference on Robotics and Automation, Roma (2007) from : http://ieeexplore.ieee.org/xpl/articleDetails.jsp?arnumb er=4209207

[6] http://www.touchbionics.com/products/activeprostheses/i-limb-ultra/

[7] http://www.touchbionics.com/products/activeprostheses/i-limb-ultra/features/

[8] Tetsuya Mouri and Haruhisa Kawasaki, A Novel Anthropomorphic Robot Hand and its Master Slave System from: http://www.intechopen.com/books/humanoid robots $\mathrm{h}$ uman_like_machines/a_novel_anthropomorphic_robot _hand_and_its_master_slave_system

[9] David J. Cassar and Michael A. Saliba "A ForceFeedback Glove based on Magnetorheological Fluid: Preliminary Design Issues" http://www.eng.um.edu.mt/ masali/publications/MEL ECON2010.pdf

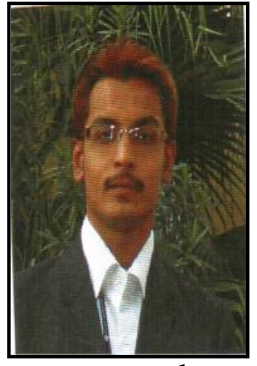

Anand Kumar Kirori S/o Shri. Pramod Kumar Kirori and Smt Madhu Kirori, was born on 14-08-1987. He completed his Bachelor of Engineering in Electronics and Communications from Rajasthan University, India in 2008. His area of interests lies in the field of Physical Human-Robot Interaction, Image Processing, Embedded System, Real time systems and many other areas. He is an M.Tech Scholar from Jaipur National University, Jaipur. He has done many projects and research work in the field of robotics.

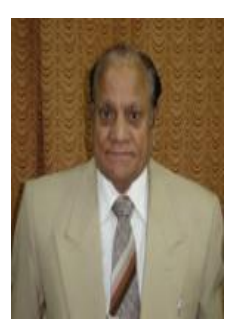

Professor Rajeshwar Lal Dua a Fellow Life Member of IETE and also a Life Member of: I.V.S. \& I.P.A. former "Scientist F" of the Central Electronics Engineering Research Institute (CEERI), Pilani has been one of the most well known scientists in India in the field of Vacuum Electronics Devices for over three and half decades. His professional achievements span a wide area of vacuum microwave devices ranging from crossed-field and linear-beam devices to present-day gyrotrons. 